\title{
Cognitive impairment and "invisible symptoms" are not associated with CCSVI in MS
}

\author{
Carmela Leone ${ }^{1}$ Emanuele D'Amico', Sabina Cilia', Alessandra Nicoletti', Luigi Di Pino² and Francesco Patti ${ }^{*}$
}

\begin{abstract}
Background: We investigated the association between chronic cerebrospinal venous insufficiency (CCSVI) and cognitive impairment (CI) in multiple sclerosis (MS). Moreover, we evaluated the association between CCSVI and other frequent self-reported MS symptoms.

Methods: We looked at the presence of Cl in incident MS patients with CCVSI in a population-based cohort of Catania, Italy. All subjects were group-matched by age, sex, disease duration and EDSS score with MS patients without CCSVI, serving as controls. Cl was assessed with the Brief Repeatable Battery (BRB) and the Stroop Test (ST) and it was defined by the presence of at least three impaired tests. Fatigue and depressive symptoms were assessed with Fatigue Severity Scale (FSS) and Hamilton Depressive Rating Scale (HDRS), respectively. Bladder and sexual symptoms were assessed with the respective items of the Italian version of Guy's Neurological Disability Scale (GNDS). Quality of life was evaluated with Multiple Sclerosis Quality of Life-54 Instrument (MSQOL-54).

Results: Out of 61 MS patients enrolled in the study, 27 were CCSVI positive and 34 were CCSVI negative. Of them, 43 were women (70.5\%); the mean age was $43.9 \pm 11.8$ years; the mean disease duration was $159.7 \pm 113.7$ months; mean EDSS was $3.0 \pm 2.6$. Of them, 36 (59.0\%) were classified relapsing-remitting (RR), 12 (19.7\%) secondary progressive (SP), seven (11.5\%) primary progressive (PP) and six (9.3\%) Clinically Isolated Syndrome (CIS). Overall, Cl was detected in 29/61 (47.5\%) MS patients; particularly 13/27 (48.1\%) in the CCSVI positive group and 16/34 (47.0\%) in the CCSVI negative group. Presence of CCSVI was not significantly associated with the presence of $\mathrm{Cl}(\mathrm{OR} 1.04 ; 95 \% \mathrm{Cl}$ 0.37-2.87; p-value $=0.9)$. Not significant differences were found between the two groups regarding the other MS symptoms investigated.
\end{abstract}

Conclusions: Our findings suggest a lack of association between CCSVI and Cl in MS patients. Fatigue, depressive, bladder/sexual symptoms and self-reported quality of life are not associated with CCSVI.

\section{Background}

Multiple sclerosis (MS) is an inflammatory-mediated demyelinating disease of the Central Nervous System (CNS), characterized also by axonal damage in the brain and spinal cord [1] and cortical lesions [2]; its pathogenesis is still unknown, but it is most likely caused by a complex interplay between polygenetic and environmental factors $[3,4]$.

Recently, it was hypothesized that extra-cranial venous abnormalities, named chronic cerebrospinal venous insufficiency (CCSVI), might play a role in the pathogenesis of MS $[5,6]$. CCSVI has been described to interfere with venous drainage from the CNS, which contributes to the development and progression of MS; moreover,

\footnotetext{
*Correspondence: patti@unict.it

'Department GF Ingrassia, Section of Neurosciences, University of Catania, Catania, Italy

Full list of author information is available at the end of the article
}

when the stenotic lesions in the internal jugular and azygos veins were treated with balloon angioplasty, rapid and often dramatic symptomatic improvement in many patients has been reported [7]. However, a number of studies have showed controversial data (ranges from $0 \%$ to $100 \%$ ) about the frequency of CCSVI in MS patients so far see in review, [8]. Recently, our group found that CCSVI was present in $18.9 \%$ of MS patients [9], but one more recent study did not find any association between CCSVI and MS [10]. Although several studies have recently evaluated the effects of CCSVI endovascular treatment on subjective aspects of MS, such as fatigue, patients clinical status and their quality-of-life [7,11-14], to date no studies have investigated the possibility of an association between CCSVI and symptoms of MS.

MS determines not only motor symptoms and signs which are responsible for the so-called physical disability,

\section{Biomed Central}

(c) 2013 Leone et al.; licensee BioMed Central Ltd. This is an Open Access article distributed under the terms of the Creative Commons Attribution License (http://creativecommons.org/licenses/by/2.0), which permits unrestricted use, distribution, and reproduction in any medium, provided the original work is properly cited. 
but it is also responsible for a number of non-motor, selfreported symptoms and signs so-called "invisible symptoms" [15]. Among them, in recent years, much attention has been focused on bladder and sexual disorders, visual symptoms, fatigue, pain, depression and anxiety, and especially, on cognitive disorders.

Cognitive impairment (CI) is a well-recognized symptom of MS, occurring in about half of all patients; it is a disabling symptom which contributes to the poor quality of life in MS patients [16]. CI is a common finding in brain vascular diseases also; however, because of evolving definitional criteria, the precise frequency of vascular CI is difficult to assess [17]. The cerebral flow reduction, which is a key feature of brain vascular diseases, has been recently investigated in MS [18]. A widespread cerebral hypo perfusion seen in MS [18], leading to brain atrophy, could be the result of the venous outflow obstructions secondary to CCSVI [19].

We aimed to investigate whether CCSVI may be associated with $\mathrm{CI}$ in MS patients. Bearing in mind that $\mathrm{CI}$ could be a direct effect of MS or an effect of the cerebrovascular hypo perfusion, we sought to investigate the presence of a possible association between CCSVI and $\mathrm{CI}$ in MS patients.

\section{Methods}

\section{Ethics statement}

The study was approved by the two different local ethical committees (Azienda Universitaria-Ospedaliera Policlinico Vittorio Emanuele di Catania and Ethical Committe of the Azienda Sanitaria Locale 3 of Catania). Patients and controls were enrolled after they had signed the informed consent.

\section{Study population}

We recently carried out a population-based case-control study to evaluate the possible association between MS and CCSVI [9]. Briefly, from 1 January 1975 to 31 December 2004, 367 MS patients resident in the study area had had the onset of disease [20-22]. Presence of CCSVI was evaluated in $148 \mathrm{MS}$ patients randomly selected from this well defined incident-cohort and in 20 patients affected by CIS. All MS subjects enrolled in the study had fulfilled Poser criteria for Clinically Defined MS [23]. All CIS subjects enrolled had fulfilled McDonald criteria [24]. CCSVI, defined according to the Zamboni's criteria, was found in 28 of the $148 \mathrm{MS}$ patients, and in 2 of the CIS patients. Details are described elsewhere [9]. To evaluate the possible association between CI and CCSVI we enrolled in the present study, all MS and CIS patients who fulfilled the diagnostic criteria for CCSVI identified in the previous population-based case-control study [9]. Control subjects were selected among the 130 patients without CCSVI and were "frequency matched" by age ( \pm five years), sex, mean
EDSS, mean disease duration and disease course. Exclusion criteria were the presence of known vascular malformations and mental illness (history of psychiatric disorders including suicidal ideation or any episode of clinically severe depression diagnosed by DSMIV [25]), history of chronic drug or alcohol abuse prior to neuropsychological (NPS) examination, any traumatic history occurring within 3 months prior to NPS examination and pregnancy. Patients who had experienced a MS relapse within the 50 days prior to study entry were excluded from the study.

\section{Clinical and exposure assessment}

As described elsewhere, the presence of CCSVI was defined as the presence of at least two out of the follow five parameters [5]:

I. Reflux in the IJV and/or VVs in sitting and supine posture;

II. Reflux in the DCVs;

III. High-resolution B-mode evidence of IJV stenosis;

IV. Flow not Doppler detectable in the IJVs and/or VVs;

$\mathrm{V}$. Reverted postural control of the main cerebral venous outflow pathways.

ECD and TCC ultrasonographies were performed by an experienced vascular sonographer who attended a course on CCSVI at the University of Ferrara in 2011 (supervisor Dr Zamboni, who first described this modality of CCSVI assessment in MS). All enrolled patients underwent a complete physical examination, including blood pressure measurement and detailed medical history; they also underwent a CT angiography to evaluate other possible causes of cerebrovascular disease.

\section{NPS assessment}

All neurological examinations were performed by trained and certified examining neurologist [Neurostatus, 2006; available at http://www.neurostatus.net]; for each patient the Expanded Disability Status Scale (EDSS) [26] was recorded the same day of NPS assessment, which was obtained within two weeks from Echo Color Doppler examination.

The NPS assessments included Rao's Brief Repeatable Battery (BRB) $[27,28]$ and the Stroop Colour-Word Task (ST) [29] for cognitive domains. All patients underwent cognitive testing for the first time and took the same form of the BRB test (Form A). The BRB incorporates tests of verbal memory acquisition and delayed recall (Selective Reminding Test, SRT) [30], visual memory acquisition and delayed recall (10/36 Spatial Recall Test, SPART) [31], attention, concentration, and speed of information processing (Paced Auditory Serial Addition Test, PASAT [32]; Symbol Digit Modalities Test, SDMT) [33], and verbal fluency on semantic stimulus (Word List 
Generation, WLG) [34]. All NPS tests were administered in the following order: SRT Long Term Storage (LTS, SRT Consistent Long-Term Retrieval (SRT-CLTR), SPART, SDMT, PASAT 3', PASAT 2', SRT-Delayed (SRT-D), SPART-Delayed (SPART-D), WLG-A and ST. All the patients were examined by the same neuropsychologist in order to uniform criteria of administration, data recording and scoring procedures. In order to guarantee the blinding of the neuropsychologist, we instructed subjects not to reveal their CCSVI status during NPS examination. Failure on a test was defined using the available normative data for the Italian population, considering the fifth percentile of the Italian population performance on each evaluation as the cut-off point for calculating the number of failed tests of the BRB; for the ST we considered the ninety-fifth percentile [27]. Cut-off points were applied to the scores adjusted by age and education [27].

Patient intelligence quotient (IQ) was determined before the NPS assessment by administering the Brief Intelligence Test (BIT) [35]. The BIT is based on the correlation between general intelligence and reading ability. The test comprises a reading skill test of 54 words with either regular or irregular emphasis (accent) [35,36].

Quality of life (QoL) was assessed by the MSQoL-54 questionnaire [37], depressive symptoms by the Hamilton Depression Rating Scale (HDRS) [38], fatigue by the Fatigue Severity Scale (FSS) [39], bladder and sexual symptoms by the respective items of the Italian version of GNDS [40]. These tests were self-administered one-three days before NPS testing.

\section{Statistical analysis}

Data were analyzed using STATA 10.0 software packages [41]. Data were double entered into the database. Data cleaning was also performed before the data analysis considering both range and consistence checks. Quantitative variables were described using mean and standard deviation (mean $\pm \mathrm{SD}$ ). Means and proportions were assessed by t-test and Chi-square test respectively. In case of not a normal distribution appropriate non-parametric tests were performed. Unconditional logistic regression analysis was performed and for each study variable, we calculated $\mathrm{OR}, 95 \% \mathrm{CI}$, and $\mathrm{p}$ value (two-tailed test, $\mathrm{p}=0.05$ ). Parameters associated with the outcome at the univariate analysis with a threshold of $p=0.10$ were included in the model. The model was manually constructed using the Likelihood Ratio Test (LRT) to compare the log-likelihood of the model with and without a specific variable.

Multiple linear regression analysis was used to test whether demographic and clinical characteristics including CCSVI, can predict the cognitive outcome. The number of failed subtests was selected as the variable representing the extent of cognitive decline.
Table 1 Baseline demographic and clinical characteristics of study sample

\begin{tabular}{lccc}
\hline & CCSVI- & CCSVI+ & p-value \\
\hline $\mathbf{N}^{\circ}(\%)$ & 34 & 27 & 0.3 \\
\hline W/M (\%) & $24(70.5)$ & $19(70.3)$ & 1.0 \\
\hline Age (mean \pm SD) & $42.3 \pm 12.3$ & $46.1 \pm 11.1$ & 0.3 \\
\hline EDSS (mean \pm SD) & $3.1 \pm 2.8$ & $3.0 \pm 2.3$ & 1.0 \\
\hline Disease Duration (years; mean \pm SD) & $13 \pm 9.9$ & $13.7 \pm 8.8$ & 0.6 \\
\hline Mean Education (years; mean \pm SD) & $11.9 \pm 3.9$ & $12.1 \pm 4.6$ & 0.7 \\
\hline RR (\%) & $19(55.9)$ & $17(63.0)$ & 0.5 \\
\hline SP (\%) & $9(26.5)$ & $3(11.1)$ & 0.2 \\
\hline PP (\%) & $2(5.9)$ & $5(18.5)$ & 0.2 \\
\hline CIS (\%) & $4(11.8)$ & $2(7.4)$ & 0.7 \\
\hline
\end{tabular}

Data are given as means \pm standard deviations.

W women.

$M$ men.

RR relapsing-remitting.

SP secondary progressive.

PP primary progressive.

CIS clinically isolated syndrome.

Whenever variables were dichotomized or polychotomized, the cut-offs were derived from the pooled distribution of cases and control subjects (e.g., using the median, tertiles, or quartiles).

\section{Results}

Out of the 30 patients (28 MS and 2 CIS) CCSVI positive identified in the previous population-based case-control study, 27 (25 MS and two CIS) were enrolled in the study. Out of the 138 MS and CIS patients without CCSVI, identified in the previous study, using a frequency matching, 34 similar in terms of age ( \pm 5 years), sex, mean EDSS, mean disease duration and disease course were enrolled in the study as control group.

Demographic and clinical characteristics of patients with and without CCSVI are reported in Table 1.

Table 2 Distribution of venous hemodynamic criteria among CCSVI positive patients

\begin{tabular}{lc}
\hline & Number of CCSVI positive patients (\%) \\
\hline Criterion III & $19(28.8 \%)$ \\
\hline Criterion II & $16(24.2 \%)$ \\
\hline Criterion I & $15(22.7 \%)$ \\
\hline Criterion V & $15(22.7 \%)$ \\
\hline Criterion IV & $1(1.5 \%)$ \\
\hline
\end{tabular}

Criterion I = Reflux in the IJVs and/or Ws in sitting and supine posture. Criterion II = Reflux in the DCVs.

Criterion III = High-resolution B-mode evidence of proximal IJV stenoses.

Criterion IV = Flow not Doppler detectable in the IJVs and/or VVs.

Criterion $\mathbf{V}=$ Reverted postural control of the main cerebral venous outflow Pathway ( $\triangle$ CSA).

$\mathrm{N}$ number.

IJVs internal jugular veins.

VVs vertebral veins.

DCVs deep cerebral veins. 
Table 3 Multiple linear regression analysis: predictors of cognitive dysfunction

\begin{tabular}{lccc}
\hline Outcome & Predictors & Coefficient & p-value \\
\hline Failed subtests & CCSVI & .02 & 0.9 \\
\cline { 2 - 4 } & Sex & -.45 & 0.4 \\
\cline { 2 - 4 } & Age & .06 & 0.01 \\
\cline { 2 - 4 } & EDSS & .20 & 0.08 \\
\hline
\end{tabular}

The most common venous abnormalities found in our patients are detailed in Table 2.

CI was present in 13/27 CCSVI positive MS-patients and in 16/34 CCSVI negative MS-patients $(48.1 \%$ and $47.0 \%$, respectively). At univariate analysis presence of CCSVI was not associated with presence of CI (OR 1.04; 95\% CI 0.37-2.87; p-value $=0.9$ ). A close association (OR 0.90; 95\% CI 0.23-3.53; p-value $=0.8$ ) was found performing a multivariate analysis and adjusting by age sex and EDSS considered as a priori confounders and included in the model regardless the p-value threshold.

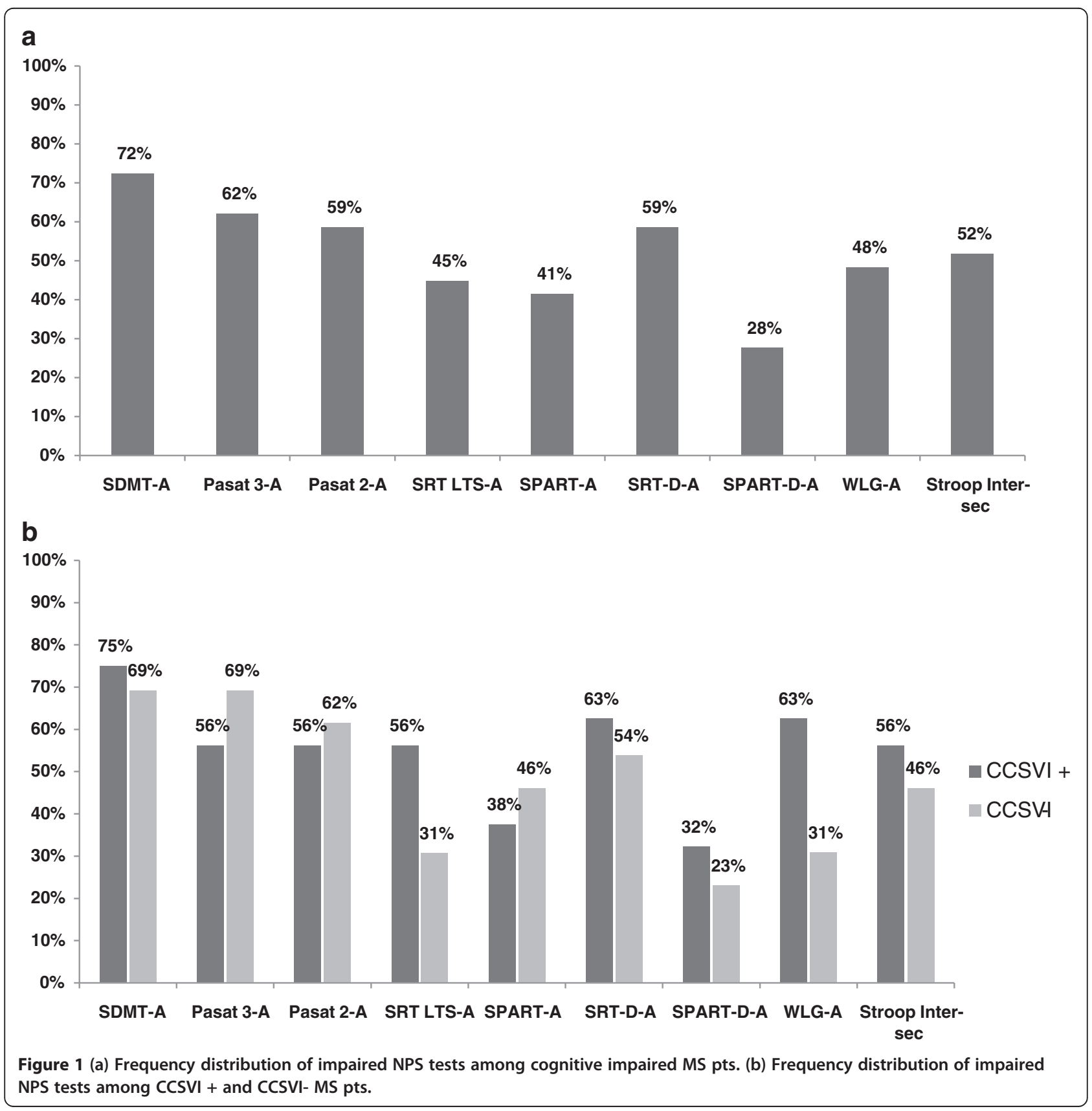


According to multiple linear regression analysis only increasing age ( $p$-value 0.01 ), followed by higher EDSS (p-value 0.08 ), proved to be positively correlated with the severity of cognitive dysfunction defined as the number of test failed as shown in Table 3.

The most frequently impaired tests found in CI patients were SDMT, 3' PASAT and 2' PASAT, SRT-D and ST (see Figure 1a). For the cognitive profile, no significant difference was found between the two groups (see Figure 1b). Regarding the self-reported measures of fatigue, depressive symptoms, bladder/sexual disturbances and quality of life not significant differences between CCSVI positive and CCSVI negative patients were found (see Table 4).

\section{Discussion}

Our study, performed in a well-defined MS population, showed that CI was not associated with CCSVI. We found about the same prevalence of CI between CCSVIpositive (48.1\%) and CCSVI-negative patients (47.0\%). Moreover, a similar prevalence of other frequent reported MS-symptoms, such as fatigue, depression and bladder/ sexual disturbances, was found between the two groups. These findings suggest that CCSVI is not correlated to the cognitive status and other MS symptoms investigated, at least in our well-selected MS population.

Our study is the first evaluating the possible association between CCSVI and one of the most disabling MS symptoms such as CI. In the few years, a number of studies were carried out to evaluate the possible association between CCSVI and MS; most of them reported higher frequency of CCSVI among MS patients and suggested a possible pathogenic role of CCSVI in determining specific symptoms and signs [5,8]. Although our recent population-based study [9] demonstrated that CCSVI was associated with MS, especially with progressive forms of the disease, there is no agreement about the exact frequency of CCSVI in MS, its putative pathogenic role and its contribution, if any, on MS symptoms
[5,8,42-45]. CI is one of the most common and disabling symptom of MS, occurring in about half of all MS patients. Literature data show that the most common cognitive deficits in MS patients are memory and speed of information processing, concentration and executive functions [16,46]. However, the mechanisms underlying CI in MS has not been fully elucidated. Several cross sectional studies demonstrated that the pathogenesis of CI in MS patients might not depend just on the extent and severity of the pathological process in the brain lesions, but also on the pathological changes affecting the normal appearing brain tissue [47-49]. Considering that, we could take into account the possible vascular hypothesis as a possible contribute to the presence of a spread or regional brain atrophy, which is one of the strongest factor correlated with the CI in MS [50-52]. Impaired cerebral perfusion seems to be related to CI [18] and a widespread cerebral hypo perfusion in MS, described as a potential cause of brain atrophy [50-52], could be the result of the venous outflow obstructions as seen in the CCSVI [19]. Although there is no strong evidence suggesting that CCSVI is a cause of MS, there is some evidence that a slower cerebral venous flow in patients with MS might be secondary to the reduced cerebral blood flow [19].

Our results do not support the hypothesis that CCSVI might be responsible for the appearance or worsening of CI in MS.

This study represents the first population-based case control-study looking at the possible association between CCSVI and CI in MS.

The population-based design represents the main strength of our study reducing the risk of a possible selection bias. Nevertheless such design has led to a small study size allowing us to enrol just a limited number of cases and controls. Consequently the lack of association between CCSVI and CI in MS patients could be due to a lack of power (type II error). Furthermore the retrospective nature of the study did not allow us to establish the exact sequence of the events. In order to exclude a

Table 4 Self-report MS symptoms measures at the time of evaluation

\begin{tabular}{lllll}
\hline & CCSVI- & CCSVI+ & p-value \\
\hline FSS (mean \pm SD) & $3.7 \pm 1.7$ & $3.0 \pm 1.8$ & 0.7 \\
\hline HDRS (mean \pm SD) & $5.0 \pm 3.7$ & $6.7 \pm 4.6$ & 0.7 \\
\hline Bladder symptoms GNDS (mean \pm SD) & $1 \pm 1.4$ & $0.8 \pm 1.0$ & 0.7 \\
\hline Sexual symptoms GNDS (mean \pm SD) & $1 \pm 1.8$ & $0.96 \pm 1.4$ & 0.6 \\
\hline MSQOL-54-PHCS (mean \pm SD) & $65.3 \pm 21.4$ & $70.3 \pm 17.2$ & 0.2 \\
\hline MSQOL-54-MHCS (mean \pm SD) & $64.2 \pm 20.4$ & $67.5 \pm 19.6$ & 0.2 \\
\hline
\end{tabular}

Data are given as means \pm standard deviations.

FSS Fatigue Severity Scale.

HDRS Hamilton depression Rating Scale.

GNDS Guy's Neurological Disability Scale.

MSQOL-54 Multiple Sclerosis Quality of Life-54.

PHCS Physical Health Composite Score.

MHCS Mental Health Composite Score. 
number of conditions which might have influenced the NPS performance, we evaluated subjective fatigue and depressive symptoms, excluding that these symptoms could be correlated with CCSVI.

CI was detected by a single trained Neuropsychologist in order to avoid any inter-rater disagreement.

Although the role of CCSVI in MS pathology is still unknown and the previous evidence are controversial so far, further prospective studies are needed to withdraw the hypothesis that CCSVI might play a role in determining $\mathrm{CI}$ or other invisible symptoms in MS.

\section{Conclusions}

Our findings suggest a lack of association between the presence of CCSVI with CI and others "invisible symptoms" in patients with MS.

\section{Abbreviations}

BRB: Rao's brief repeatable battery; CCSVI: Chronic cerebrospinal venous insufficiency; Cl: Cognitive impairment; CIS: Clinically isolated syndrome; EDSS: Expanded disability status scale; FSS: Fatigue severity scale; GNDS: Guy's neurological disability scale; HDRS: Hamilton depressive rating scale; MR: Magnetic resonance; MS: Multiple sclerosis;

NPS: Neuropsychological; OR: Odds ratio; RR: Relapsing-remitting; SP: Secondary progressive; PP: Primary progressive.

\section{Competing interests}

\section{Financial competing interests}

PF has received honoraria for speaking activities by Bayer Schering, Biogen Idec, Merck Serono, Novartis and Sanofi Aventis; he also served as advisory board member the following companies: Bayer Schering, Biogen Idec, Merck Serono, Novartis; he was also funded by Pfeizer and FISM for epidemiological studies; finally he received grant for congress participation from Bayer Schering, Biogen Idec, Merck Serono, Novartis, Sanofi Aventis and TEVA. LC received partial grant for congress participation from Bayer Schering and Biogen Idec. DAE received grants for congress participation from Sanofi Aventis. CS declares that she has not competing interests. NA declares that she has not competing interests. DPL declares that he has not competing interests.

\section{Non financial competing interests}

This study was not sponsored even if RELOAD ONLUS supported the study, providing the tilt table used in the study.

\section{Authors' contributions}

LC participated in the design of the study and in manuscript preparation. DAE performed neurological examination of patients. CS performed neuropsychological examination of patients. NA participated in the study design, manuscript preparation and performed the statistical analyses. DPL carried out the ECD and TCC evaluations. PF conceived the study and participated in its design and coordination. All authors read and approved the final manuscript.

\section{Acknowledgements}

The authors acknowledge RELOAD ONLUS association for partial support of the study.

\section{Funding}

This study was not sponsored even if RELOAD ONLUS supported the study, providing the tilt table used in the study.

\section{Author details}

'Department GF Ingrassia, Section of Neurosciences, University of Catania, Catania, Italy. ${ }^{2}$ Department of Cardiology, University of Catania, Catania, Italy.
Received: 2 May 2013 Accepted: 15 May 2013

Published: 27 July 2013

\section{References}

1. Compston A, Coles A: Multiple sclerosis. Lancet 2008, 372:1502-1517.

2. Calabrese M, Rinaldi F, Grossi P, Gallo P: Cortical pathology and cognitive impairment in multiple sclerosis. Expert Rev Neurother 2011, 11:425-432.

3. Mechelli R, Annibali V, Ristori G, Vittori D, Coarelli G, Salvetti M: Multiple sclerosis etiology: beyond genes and environment. Expert Rev Clin Immunol 2010, 6:481-490.

4. Goverman J: Autoimmune T, cell responses in the central nervous system. Nat Rev Immunol 2009, 9:393-407.

5. Zamboni P, Galeotti R, Menegatti E, Malagoni AM, Tacconi G, Dall'Ara S, Bartolomei I, Salvi F: Chronic cerebrospinal venous insufficiency al in patients with multiple sclerosis. J Neurol Neurosurg Psychiatr 2009, 80:392e9.

6. Zamboni P, Galeotti R, Menegatti E, Malagoni AM, Tacconi G, Dall'Ara S, Bartolomei I, Salvi F: The value of cerebral Doppler venous hemodynamics in the assessment of multiple sclerosis. J Neurol Sci 2009, 282:21-27.

7. Zamboni P, Galeotti R, Menegatti E, Malagoni AM, Gianesini S, Bartolomei I, Mascoli F, Salvi F: A prospective open-label study of endovascular treatment of chronic cerebrospinal venous insufficiency. J Vasc Surg 2009, 50:1348-1358

8. Laupacis A, Lillie E, Dueck A, Straus S, Perrier L, Burton JM, Aviv R, Thorpe K, Feasby T, Spears J: Association between chronic cerebrospinal venous insufficiency and multiple sclerosis: a meta-analysis. CMAJ 2011, 183:1203-1212.

9. Patti F, Nicoletti A, Leone C, Messina S, D’Amico E, Lo Fermo S, Paradisi V, Bruno E, Quattrocchi G, Veroux P, Di Pino L, Costanzo L, Zappia M: Multiple sclerosis and CCSVI: a population-based case control study. PLoS One 2012, 7:e41227.

10. Comi G, Battaglia MA, Bertolotto A, Del Sette M, Ghezzi A, Malferrari G, Salvetti M, Sormani M, Stolz E, Tesio L, Mancardi GL, on behalf of the CoSMo study group: Chronic Cerebro-Spinal Venous Insufficiency (CCSVI) and global venous haemodynamics in multiple sclerosis: the CoSMo study. Mult Scler 2012, 18(4 suppl):509-520.

11. Malagoni AM, Galeotti R, Menegatti E, Manfredini F, Basaglia N, Salvi F, Zamboni P: Is chronic fatigue the symptom of venous insufficiency associated with multiple sclerosis? A longitudinal pilot study. Int Angiol 2010, 29:176-182.

12. Ludyga T, Kazibudzki M, Latacz P, Świerad M, Piegza J, Hartel M, Kluczewska E, Simka M: Early results of a prospective open-label study on endovascular treatments for chronic cerebrospinal venous insufficiency in the patients with associated multiple sclerosis. Phlebol Rev 2011, 19:9-14.

13. Kostecki J, Zaniewski M, Ziaja K, Urbanek T, Kuczmik W, Krzystanek E, Ziaja D, Korzeniowski T, Majewski E, Hartel M, Swiat M, Sioma-Markowska U: An endovascular treatment of chronic Cerebro-spinal venous insufficiency in multiple sclerosis patients -6 month follow-up results. Neuro Endocrinol Lett 2011, 32:557-562.

14. Simka M, Janas $P$, Ludyga $T$, Latacz $P$, Kazibudzki M: Endovascular treatment for chronic Cerebro-spinal venous insufficiency in patients with multiple sclerosis. Vasc Dis Manage 2012, 9:E149-E154.

15. White CP, White MB, Russel CS: Invisible and visible symptoms of multiple sclerosis: which are more predictive of health distress. $J$ Neurosci Nurs 2008, 40(2):85-88.

16. Patti F: Cognitive impairment in multiple sclerosis. Mult Scler 2009, 15:2-8.

17. Selnes OA, Vinters HV: Vascular cognitive impairment. Nat Clin Pract Neurol 2006, 2:538-547.

18. D'haeseleer M, Cambron M, Vanopdenbosch L, De Keyser J: Vascular aspects of multiple sclerosis. Lancet Neurol 2011, 10:657-666.

19. Singh AV, Zamboni P: Anomalous venous blood flow and iron deposition in multiple sclerosis. J Cereb Blood Flow Metab 2009, 29:1867e78.

20. Nicoletti A, Lo Bartolo ML, Lo Fermo S, et al: Prevalence and incidence of multiple sclerosis in Catania, Sicily. Neurology 2001, 56(1):62-66.

21. Nicoletti A, Patti F, Lo Fermo $S$, et al: Possible increasing risk of multiple sclerosis in Catania, Sicily. Neurology 2005, 65(8):1259-1263.

22. Nicoletti A, Patti F, Lo Fermo S, et al: Increasing frequency of multiple sclerosis in Catania, Sicily: a 30-year survey. Mult Scler 2011, 17(3):273-280.

23. Poser CM, Paty DW, Scheinberg L, McDonald WI, Davis FA, Ebers GC, Johnson KP, Sibley WA, Silberberg DH, Tourtellotte WW: New diagnostic 
criteria for multiple sclerosis: guidelines for research protocols. Ann Neurol 1983, 13:227-231.

24. Mc Donald WI, Compston A, Edan G, Goodkin D, Hartung HP, Lublin FD, McFarland HF, Paty DW, Polman CH, Reingold SC, Sandberg-Wollheim M, Sibley W, Thompson A, van den Noort S, Weinshenker BY, Wolinsky JS: Recommended diagnostic criteria for multiple sclerosis: guidelines from the international panel on the diagnosis of multiple sclerosis. Ann Neurol 2001, 51:121-127.

25. American Psychiatric Association: Diagnostic and statistical manual of mental disorders, fourth edition, text revision. 4th edition. Washington, DC: American Psychiatric Association; 2000.

26. Kurtzke JF: Rating neurologic impairment in multiple sclerosis: an expanded disability status scale (EDSS). Neurology 1983, 33:1444-1452.

27. Amato MP, Portaccio E, Goretti B, Zipoli V, Ricchiuti L, De Caro MF, Patti F, Vecchio R, Sorbi S, Trojano M: The Rao's Brief repeatable battery and Stroop test: normative values with age, education and gender corrections in an Italian population. Mult Scler 2006, 12:787-793.

28. Boringa JB, Lazeron RH, Reuling IE, Adèr HJ, Pfennings L, Lindeboom J, de Sonneville LM, Kalkers NF, Polman CH: The brief repeatable battery of neuropsychological tests: normative values allow application in multiple sclerosis clinical practice. Mult Scler 2001, 7:263-267.

29. Denney DR, Lynch SG: The impact of multiple sclerosis on patients' performance on the Stroop Test: processing speed versus interference. $J$ Int Neuropsychol Soc 2009, 15:451-458.

30. Buschke H, Fuld PA: Evaluating storage, retention, and retrieval in disordered memory and learning. Neurology 1974, 24:1019-1025.

31. Barbizet J, Cany E: Clinical and psychometrical study of a patient with memory disturbances. Int J Neurol 1968, 7:44-54.

32. Gronwall DM: Paced auditory serial-addition task: a measure of recovery from concussion. Percept Mot Skills 1977, 44:367-373.

33. Smith A: Symbol digit modalities test. Los Angeles: Western Psychological Services; 1991

34. Rao SM, Cognitive Function Study Group of the National Multiple Sclerosis Society: A manual for the brief repeatable battery of neuropsychological tests in multiple sclerosis. Milwaukee, WI: Medical College of Wisconsin; 1990

35. Kaufman AS, Wang JJ: Gender, race, and education differences on the KBit at ages 4 to 90 years. J Psychoeducational Assess 1992, 10:219-229.

36. Colombo L, Sartori G, Brivio C: La stima del quoziente intellettivo tramite I'applicazione del TIB (Test di Intelligenza Breve). G Ital Psicol 2002, 3:613-637.

37. Vickrey BG, Hays RD, Harooni R, Myers LW, Ellison GW: A health-related quality of life measure for multiple sclerosis. Qual Life Res 1995, 4(3):187-206.

38. Hamilton M: Development of a rating scale for primary depressive illness. Br J Soc Clin Psychol 1967, 6:278-296.

39. Fisk JD, Ritvo PG, Ross L, Haase DA, Marrie TJ, Schlech WF: Measuring the functional impact of fatigue: initial validation of the fatigue impact scale. Clin Infect Dis 1994, 18(Suppl 1):S79-S83.

40. Pappalardo A, Patti F: Psychometric properties of the Italian version of the Guy's neurological disability scale. Funct Neurol 2010, 25:223-233.

41. StataCorp: Stata Statistical Software: Release 10. College Station, TX StataCorp LP; 2007.

42. Krogias C, Schroder A, Wiendl H, Hohlfeld R, Gold R: "Chronic cerebrospinal venous insufficiency" and multiple sclerosis: critical analysis and first observation in an unselected cohort of MS patients. Nervenartz 2010, 81:740-746.

43. Simka M, Kostecki J, Zaniewski M, Majewski E, Hartel M: Extra cranial Doppler sonographic criteria of chronic cerebrospinal venous insufficiency in the patients with multiple sclerosis. Int Angiol 2010, 29:109-114

44. Al-Omari MH, Rousan LA: Internal jugular vein morphology and hemodynamics in patients with multiple sclerosis. Int Angiol 2010, 29:115-120

45. Sundstrom $P$, Wahlin A, Ambarki K, Birgander R, Eklund A, Malm J: Venous and cerebrospinal fluid flow in multiple sclerosis: a case-control study. Ann Neurol 2010, 68:255-259.

46. Amato MP, Zipoli V, Portaccio E: Multiple sclerosis-related cognitive changes: a review of cross-sectional and longitudinal studies. J Neurol Sci 2006, 245:41-46.

47. Filippi M, Tortorella C, Rovaris M, Bozzali M, Possa F, Sormani MP, lannucci G, Comi G: Changes in the normal appearing brain tissue and cognitive impairment in multiple sclerosis. J Neurol Neurosurg Psychiatr 2000, 68:157-161.
48. Rovaris M, Filippi M, Falautano M, Minicucci L, Rocca MA, Martinelli V, Comi G: Relation between MR abnormalities and patterns of cognitive impairment in multiple sclerosis. Neurology 1998, 50:1601-1608.

49. Fulton JC, Grossman Rl, Udupa J, Mannon L, Grossman M, Wei L, Polansky M, Kolson DL: MR lesion load and cognitive function in patients with relapsingremitting multiple sclerosis. AJNR Am J Neuroradiol 1999, 20:1951-1955.

50. Benedict RH, Carone DA, Bakshi R: Correlating brain atrophy with cognitive dysfunction, mood disturbances, and personality disorder in multiple sclerosis. J Neuroimaging 2004, 14:36S-45S.

51. Sanchez MP, Nieto A, Barroso J, Martín V, Hernández MA: Brain atrophy as a marker of cognitive impairment in mildly disabling relapsing-remitting multiple sclerosis. Eur J Neurol 2008, 15:1091-1099.

52. Calabrese M, Agosta F, Rinaldi F, Mattisi I, Grossi P, Favaretto A, Atzori M, Bernardi V, Barachino L, Rinaldi L, Perini P, Gallo P, Filippi M: Cortical lesions and atrophy associated with cognitive impairment in relapsing-remitting multiple sclerosis. Arch Neurol 2009, 66:1144-1150.

doi:10.1186/1471-2377-13-97

Cite this article as: Leone et al:: Cognitive impairment and "invisible symptoms" are not associated with CCSVI in MS. BMC Neurology 2013 13:97

\section{Submit your next manuscript to BioMed Central and take full advantage of:}

- Convenient online submission

- Thorough peer review

- No space constraints or color figure charges

- Immediate publication on acceptance

- Inclusion in PubMed, CAS, Scopus and Google Scholar

- Research which is freely available for redistribution 